\title{
MINIATURE STICK-PACKAGING - AN INDUSTRIAL TECHNOLOGY FOR PRE-STORAGE AND RELEASE OF REAGENTS IN LAB-ON- A-CHIP SYSTEMS
}

\author{
Thomas van Oordt ${ }^{1 *}$, Yannick Barb ${ }^{1}$, Roland Zengerle ${ }^{1,2,3}$ and Felix von Stetten ${ }^{1,2}$ \\ ${ }^{1} H S G$-IMIT, Wilhelm-Schickard-Straße 10, 78052 Villingen-Schwenningen, GERMANY \\ ${ }^{2}$ Laboratory for MEMS Applications, Department of Microsystems Engineering - IMTEK, \\ University of Freiburg, Georges-Koehler-Allee 106, 79110 Freiburg, GERMANY \\ ${ }^{3}$ BIOSS - Center for Biological Signalling Studies, University of Freiburg, GERMANY
}

\begin{abstract}
Stick-packaging of goods in tubular shaped composite-foil pouches has become a popular technology for food packaging. We miniaturized stick-packaging for use in Lab-on-a-Chip (LOAC) systems to pre-store and release liquid and dry reagents in a volume range of $\mathbf{8 0}-\mathbf{5 0 0} \boldsymbol{\mu l}$. A frangible seal integrated in the package allows for the pressure-controlled release of reagents, reducing the number of downstream valves required for liquid control. The frangible seal is fabricated by ultrasonic welding, enabling adjustment of burst pressures from 20 to $100 \mathrm{kPa}$, and allowing for packaging of temperature sensitive reagents. As an additional advantage, stick-packaging is also a scalable technology suitable for both rapid prototyping and low-cost mass production.
\end{abstract}

\section{KEYWORDS: reagent pre-storage, stick-packaging, centrifugal lab-on-a-chip}

\section{INTRODUCTION}

Today's liquid handling in microfluidic systems relies on either time-consuming manual pipetting or highly integrated but large and immobile pipetting robots. In the field of mobile analytical applications, on-chip reagent storage systems are required. The encapsulation of liquids in glass tubes has been demonstrated but it requires additional actuators to release the liquid [1]. Ideally, a test carrier based reagents storage approach will need no additional actuators for reagent release [2]. Furthermore scalability from prototyping to mass production is a further requirement. We investigated stick-packs as promising candidate for reagent pre-storage and release on a centrifugal microfluidic platform.

\section{PRINCIPLE}

An aluminium / polyethylene composite foil (standard stickpack film12/7/40, Safta S.p.A., Italy) is processed on an industrial packaging machine (GVL, Germany) into $5-7 \mathrm{~mm}$ wide tubular packages (stick-packs) of variable length (Figure 1a). These downsized stick-packs are equipped with two different types of sealing: one strongly bonded longitudinal seal and two weakly bonded transverse seals. One of the transverse seals is used as a frangible seal which can be adjusted to a certain burst pressure by a specific ultrasonic welding process. Ultrasonic welding is performed with a $35 \mathrm{kHz}$ welder (ECO35, Sonotronic, Germany). Fast ultrasonic welding (typically $250 \mathrm{~ms}, 12 \mu \mathrm{m}$ amplitude and $150 \mathrm{MPa}$ ) minimizes the thermal load, enabling the packaging of temperature-sensitive reagents. The hermetically sealed, miniature stick-packs can be used to store liquid or dried reagents. When inserted into a centrifugal microfluidic LOAC platform (Figure 2) the frangible seal can be adjusted to allow release of reagents by centrifugal forces at a defined burst frequency (Figure $1 \mathrm{~b}-\mathrm{c}$ ). This approach circumvents the need of any additional actuators and reduces the manufacturing costs of the processing device.
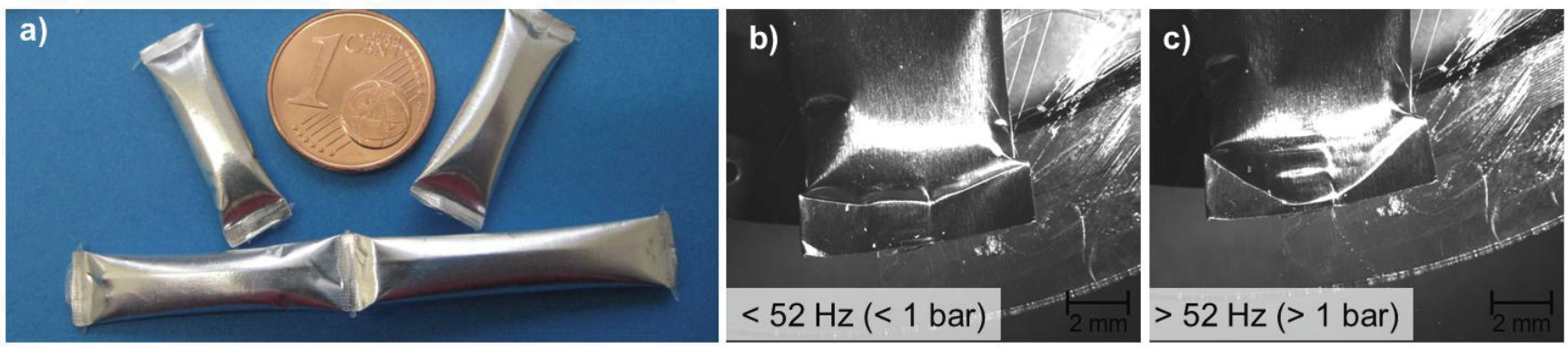

Figure 1: a) Miniature stick-packs of $7 \mathrm{~mm}$ width filled with reagents. Transverse frangible seals are bonded by ultrasonic welding and adjusted to a defined burst pressure. b) frangible seal before, and c) after release of liquid reagents upon centrifugation. 


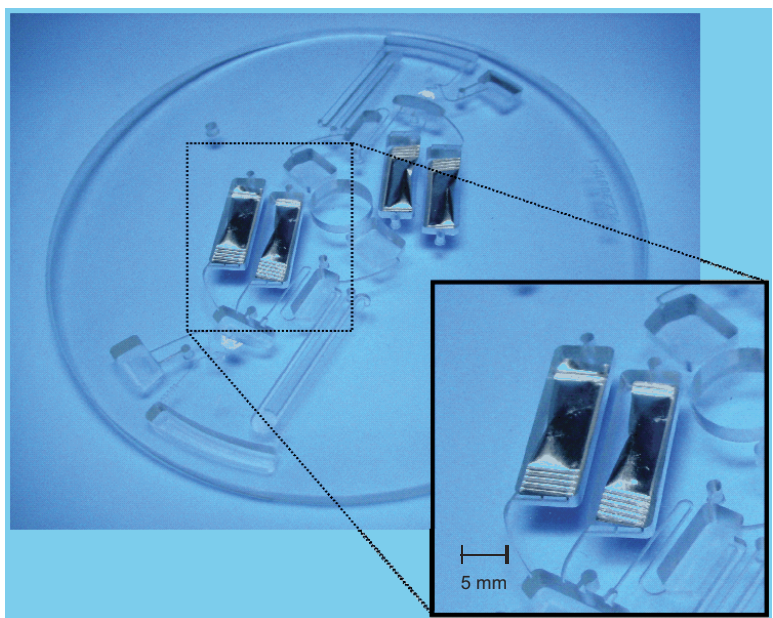

Figure 2: Photograph of a centrifugal LabDisk containing microfluidic structures for DNA-purification. The LabDisk contains miniature stick-packs containing the required washing buffers. Frangible seals allow reagent release at various predefined burst frequencies.

\section{EXPERIMENTAL}

The experimental investigation of the miniaturized stick-packs includes (a) the study of the relation between bond strength of the frangible seal and burst pressure, (b) recovery tests of the released reagents, (c) tests for liquid loss during long-term storage, (d) tests for sufficient stability of the frangible seal when pressure loss occurs during air transportation, and (e) investigation of combined storage of liquid and dry reagents with an integrated unit operation for rehydration and release.

(a) Burst pressure. Figure 3 shows how the burst pressure of a frangible seal can be adjusted by changing the sealing protocol of the ultrasonic welding machine. We found that the strength of the seal primarily depends on the amplitude of the ultrasonic horn (variation from $8-15 \mu \mathrm{m}$ ) and the sealing time (variation from $250-600 \mathrm{~ms}$ ).

(b) Recovery: At a centrifugal burst pressure of $10 \mathrm{MPa}$ liquid recovered from the stick-pack was $98 \%$ (STDV $4 \%, \mathrm{n}=$ $10,250 \mu \mathrm{l}$ water). A pressure of $40 \mathrm{MPa}$ resulted in a higher recovery of $99 \%$, STDV $1 \%$.

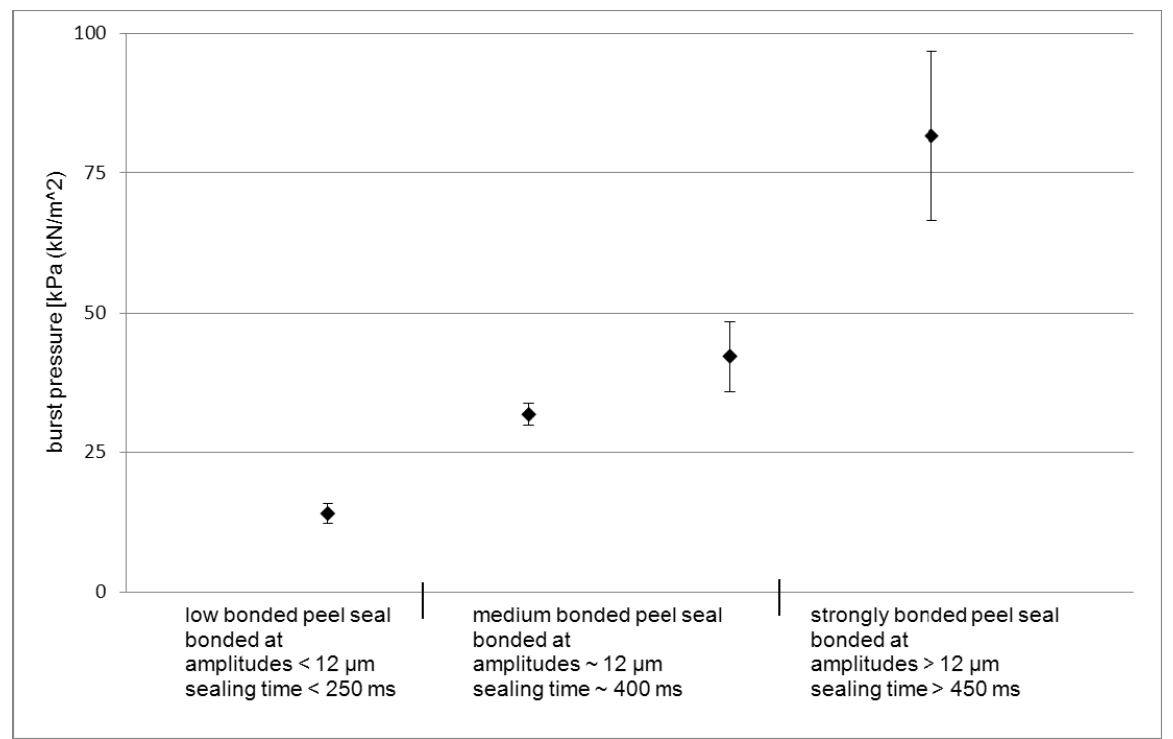

Figure 3: Burst pressure of different stick-packs filled with liquid reagents. The burst pressures of the frangible seals are adjusted by changing the sealing protocol of an ultrasonic welding machine.

(c) Long-term storage. The vapour barrier properties of the $9 \mu \mathrm{m}$ aluminium layer of the stick-pack make it suitable for longterm storage. Accelerated long-term tests $\left(250 \mu 1\right.$ water stored at $70{ }^{\circ} \mathrm{C}$ for 42 days; equivalent to $\sim 2$ years at ambient temperature) indicate that there is no significant loss of liquid due to diffusion or evaporation (loss of $0.4 \%$; $\mathrm{CV}: 1.5, \mathrm{n}=9$ ). 
(d) Air transportability. Further storage tests include the simulation of air transportation. Even seals that burst at low pressures must withstand pressure and temperature fluctuations during conventional transportation. Stick-packs were tested for 49 hours applying a pressure protocol ranging from 68 to $100 \mathrm{MPa}$ and temperature settings ranging from 25 to $55^{\circ} \mathrm{C}$ (standard IATA flight simulation test). No significant loss of liquid in a stick-pack (sealed with a "medium bonded peel seal" according to Figure 3) was observed during the entire transport simulation test (tests with a "low-bonded peel" seal are pending).

(e) Combined storage, rehydration and release of dry and liquid reagents. In addition to containing liquid, a stick-pack might store dry reagents as well. This has been tested with a combination of an aqueous buffer solution and dry skimmed milk powder. Figure 4 shows a stick-pack with two compartments: an aqueous solution in one part of the stick-pack that is separated by a frangible seal from the second part containing the dry reagent. At a defined rotational frequency the frangible seal bursts and the content of the two parts mix together. After mixing the rotational frequency of the LOAC system is increased and the second frangible seal burst and releases the freshly prepared skimmed milk solution from the stick-pack into the downstream microfluidic system. Rehydrated skim milk solutions are used for example in antibody-based assays to block surfaces and avoid unspecific binding. Many other combinations of liquid and dry reagents can be pre-stored in the same way. In our system the first frangible seal bursts at $24 \mathrm{MPa}$ (STDV $5 \mathrm{MPa}, \mathrm{n}=10$ ). The rehydrated solution is then released through the second frangible seal at a burst pressure of $66 \mathrm{MPa}$ (STDV $18 \mathrm{MPa}, \mathrm{n}=10$ ). By using dry milk in agglomerated granular form a fully solved solution is obtained even without additional mixing of the two reagents.

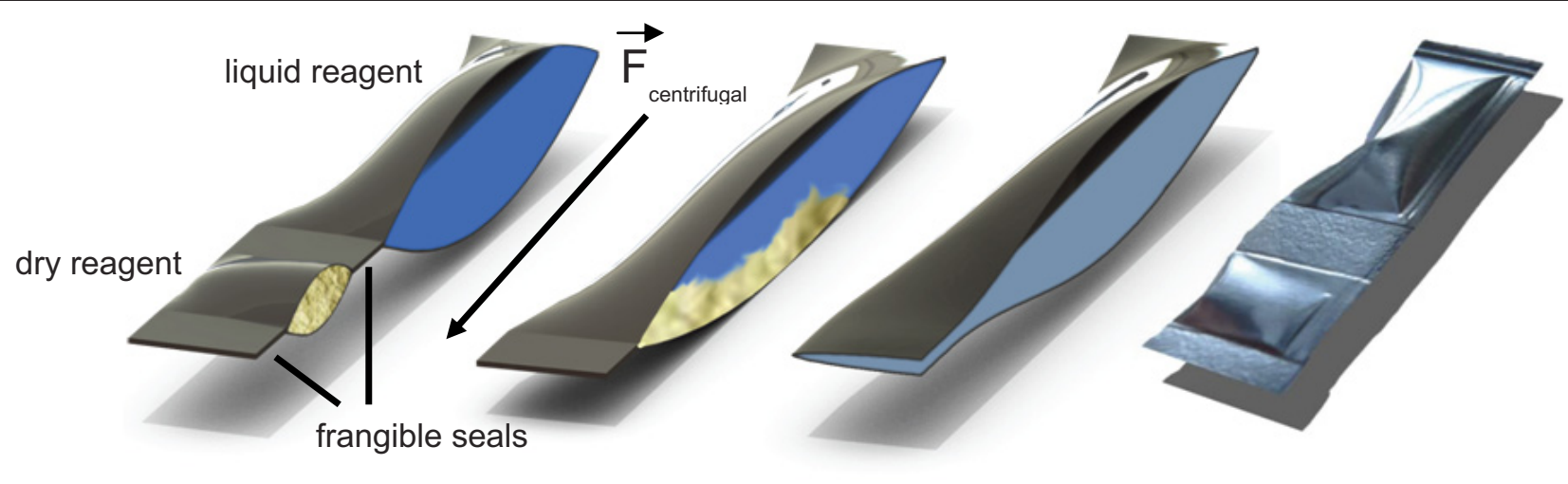

(A)

(B)

(C)

(D)

Figure 4: Miniature stick packs for combined storage, rehydration and release of dry and liquid reagents. (A) - (C): Animation of the combined storage of liquid and dry reagents in one single stick-pack. Both reagents are separated by a frangible seal (A) that bursts at a defined pressure (B). After mixing the second frangible seal burst and releases the solution (C). (D): Photograph of the stick-pack. The frangible seal that separates the two containments (the seal in the middle) bursts at a pressure of $\sim 24 \mathrm{MPa}$, the second external seal burst at a pressure of $\sim 66 \mathrm{MPa}$.

\section{CONCLUSION AND OUTLOOK}

The chip-integrated storage and release of liquid and dry reagents is an important feature in LOAC technology. Our novel approach of miniature stick-packaging combines the advantages of long-term storage of liquid and dry reagents in composite polymer pouches with an integrated rehydration and release mechanism that is triggered by adjustable burst pressures of frangible seals (e.g. by applying centrifugal force). As a scalable technology suitable for both, prototyping and massproduction, miniature stick-packaging of reagents is one of the technical advances that may help to ease the market entry of fully integrated LOAC systems.

\section{ACKNOWLEDGEMENT}

We gratefully acknowledge financial support by the Federal Ministry of Education and Research (BMBF) under the Research Programme for Civil Security of the German Federal Government as part of the High-Tech Strategy for Germany.

\section{REFERENCES}

[1] J. Hoffmann, D. Mark, S. Lutz, R. Zengerle and F. von Stetten, Lab Chip, 2010, vol. 10, pp. 1480-1484

[2] E. Guilera-Herrador, M. Cruz-Vera and M. Valcarcel, Analyst, 2010, vol. 135 (9), pp. 2220-2232

\section{CONTACT}

*T. van Oordt tel: +49-761-2038528, thomas.van.oordt@hsg-imit.de 